ARTICLE

Received 12 Jun 2014 | Accepted 2 Dec 2014 | Published 14 Jan $2015 \quad$ DOl: 10.1038/ncomms7025

\title{
Activating mutations of STAT5B and STAT3 in lymphomas derived from $\gamma \delta$-T or NK cells
}

Can Küçük ${ }^{1, \star}$, Bei Jiang ${ }^{1, \star}$, Xiaozhou Hu', Wenyan Zhang ${ }^{2}$, John K.C. Chan ${ }^{3}$, Wenming Xiao ${ }^{4}$, Nathan Lack ${ }^{5}$, Can Alkan ${ }^{6}$, John C. Williams ${ }^{7}$, Kendra N. Avery ${ }^{7}$, Pınar Kavak ${ }^{8}$, Anna Scuto ${ }^{1}$, Emel Sen ${ }^{5}$, Philippe Gaulard ${ }^{9}$, Lou Staudt ${ }^{10}$, Javeed lqbal ${ }^{11}$, Weiwei Zhang ${ }^{11}$, Adam Cornish ${ }^{12}$, Qiang Gong ${ }^{13}$, Qunpei Yang ${ }^{2}$, Hong Sun ${ }^{2}$, Francesco d'Amore ${ }^{14}$, Sirpa Leppä ${ }^{15}$, Weiping Liu ${ }^{2}$, Kai Fu ${ }^{2}$, Laurence de Leval ${ }^{16}$, Timothy McKeithan ${ }^{1}$ \& Wing C. Chan ${ }^{1}$

Lymphomas arising from NK or $\gamma \delta$-T cells are very aggressive diseases and little is known regarding their pathogenesis. Here we report frequent activating mutations of STAT3 and STAT5B in NK/T-cell lymphomas $(n=51), \gamma \delta$-T-cell lymphomas $(n=43)$ and their cell lines $(n=9)$ through next generation and/or Sanger sequencing. STAT5B N642H is particularly frequent in all forms of $\gamma \delta$-T-cell lymphomas. STAT3 and STAT5B mutations are associated with increased phosphorylated protein and a growth advantage to transduced cell lines or normal NK cells. Growth-promoting activity of the mutants can be partially inhibited by a JAK1/2 inhibitor. Molecular modelling and surface plasmon resonance measurements of the $\mathrm{N} 642 \mathrm{H}$ mutant indicate a marked increase in binding affinity of the phosphotyrosine-Y 699 with the mutant histidine. This is associated with the prolonged persistence of the mutant phosphoSTAT5B and marked increase of binding to target sites. Our findings suggest that JAK-STAT pathway inhibition may represent a therapeutic strategy.

\footnotetext{
${ }^{1}$ Department of Pathology, City of Hope Medical Center, Duarte, California 91010, USA. ${ }^{2}$ Department of Pathology, West China Hospital of Sichuan University, Chengdu 610041, China. ${ }^{3}$ Department of Pathology, Queen Elizabeth Hospital, Hong Kong, China. ${ }^{4}$ Division of Bioinformatics and Biostatistics, National Center for Toxicological Research, Food and Drug Administration, Maryland 20993, USA. ${ }^{5}$ Department of Pharmacology, Koc University, Istanbul 34450, Turkey. ${ }^{6}$ Department of Computer Engineering, Bilkent University, Ankara 06800, Turkey. ${ }^{7}$ Department of Molecular Medicine, Beckman Research Institute of City of Hope, Duarte, California 91010, USA. ${ }^{8}$ Department of Computer Engineering, Boğaziçi University, İstanbul 34342, Turkey. ${ }^{9}$ Département de Pathologie, Groupe Henri-Mondor Albert-Chenevier, Inserm U955, Université Paris Est, Créteil 94000, France. ${ }^{10}$ Molecular Biology of Lymphoid Malignancies Section, Center for Cancer Research, National Cancer Institute, Bethesda, Maryland 20892, USA. ${ }^{11}$ Department of Pathology and Microbiology, University of Nebraska Medical Center, Omaha, Nebraska 68198-3135, USA. ${ }^{12}$ Department of Genetics, Cell Biology and Anatomy, University of Nebraska Medical Center, Omaha, Nebraska 68198-5805, USA. ${ }^{13}$ Beijing Institute of Genomics, Chinese Academy of Sciences, Beijing 100029 , China. ${ }^{14}$ Department of Hematology, Aarhus University Hospital, Aarhus 8000, Denmark. ${ }^{15}$ Department of Oncology, Helsinki University Central Hospital, PO Box 180 , Helsinki 00029, Finland. ${ }^{16}$ Pathologie Clinique Institut, Universitaire de Pathologie rue du Bugnon 25, CH 1011 Lausanne, Switzerland. * These authors contributed equally to this work. Correspondence and requests for materials should be addressed to W.C.C. (email: jochan@coh.org).
} 
M ature NK-cell lymphomas are mostly classified as extranodal NK/T-cell lymphomas of nasal type (NKTCLs) $^{1}$ in the World Health Organization classification. $70-90 \%$ of NKTCLs are of NK cell lineage with the rest of T-cell origin, most of which are of the $\gamma \delta$ type. In current clinical practice, the cases are often lumped together as NKTCL because of similar clinicopathological features and management. NKTCL has poor prognosis, particularly in advanced stage or with extranasal presentation ${ }^{2}$.
In this study, we investigate the genome-wide driver mutations in NKTCLs using a combination of RNA sequencing,wholeexome sequencing (WES) and Sanger sequencing, and identify activating mutations of STAT3 and STAT5B in the Src homology 2 (SH2) domain. These mutations are also present at a high frequency $(34.9 \%)$ in $\gamma \delta$-T-cell-derived lymphomas ( $\gamma \delta$-PTCLs) and associated with promotion of growth, which can be partially inhibited by JAK1/2 inhibitors, suggesting a potential therapeutic option for these patients.

a
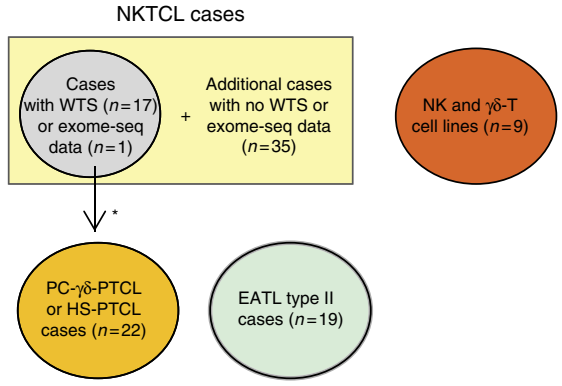

Sanger sequencing of STAT3 or STAT5B $\mathrm{SH} 2$ domains

b
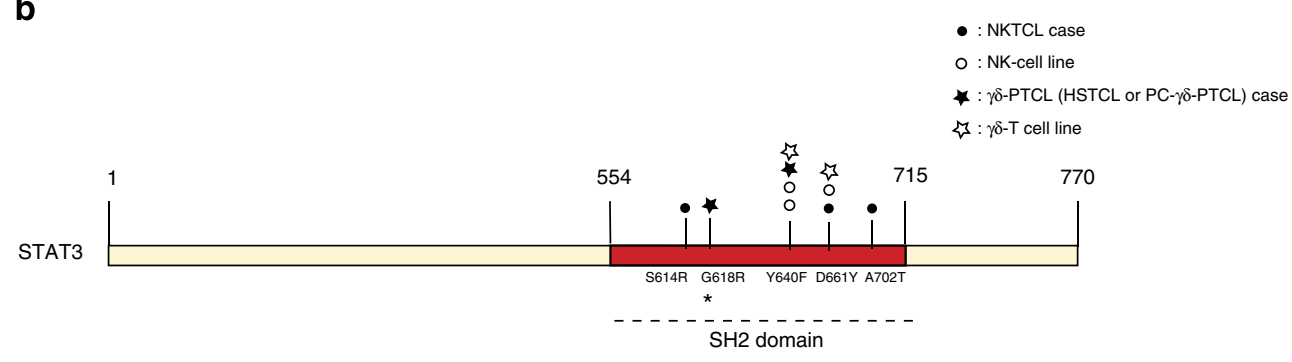

C

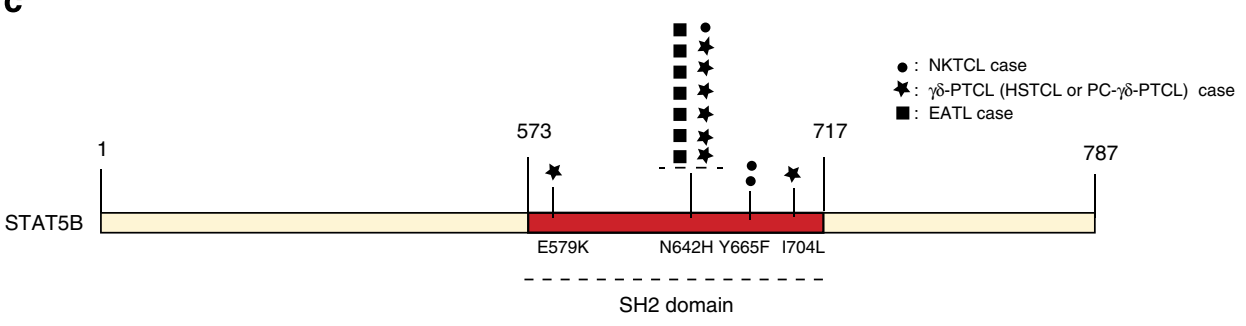

d
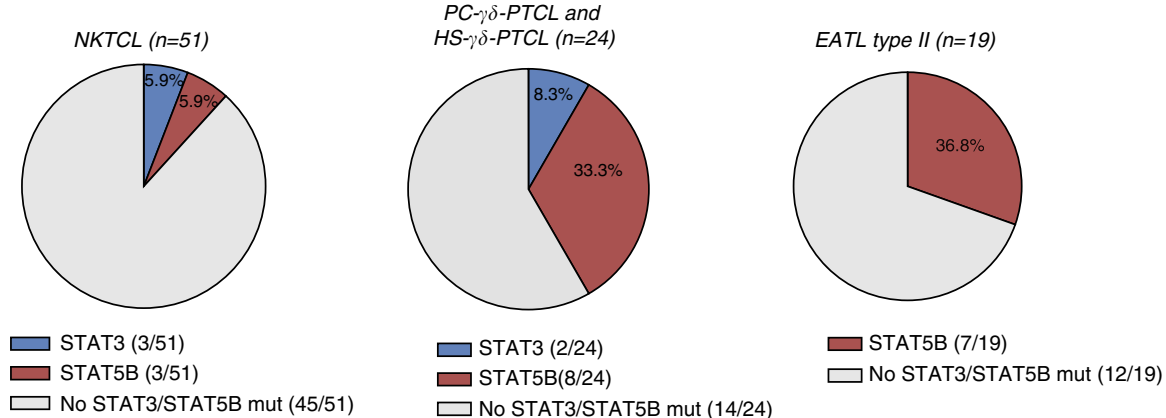

Figure 1 | Activating STAT3 and STAT5B mutations are frequent in lymphomas of NK or $\boldsymbol{\gamma} \boldsymbol{\delta}$-T cell of origin. (a) The diagram shows the type of mutation analyses performed and the number of tumour samples used for each analysis. The locations of the primers used for PCR and Sanger sequencing of the STAT3 and STAT5B SH2 domains are shown in Supplementary Fig. 1. The location of the mutated nucleotides found in the SH2 domains of STAT3 (b) and STAT5B (c) in tumour cases (NKTCL, $\gamma \delta$-PTCL (PC- $\gamma \delta$-PTCL and HS- $\gamma \delta$-PTCL) or EATL type II) and cell lines (NK and $\gamma \delta$-T cell lines) are shown (not to scale). Mutations of different tumour cases or cell lines are indicated with different symbols over the $\mathrm{SH} 2$ domains, and the disease types to which these symbols refer are shown in the upper right corner of the panels. Other than A702T, all STAT3 mutations were reported in LGLL by Koskela et al. ${ }^{27}$ and Jerez et al. ${ }^{26}$ STAT5B N642H and Y665F mutations were rarely ( $~ 1 \%)$ observed in LGLL by Rajala et al. ${ }^{28}$ (d) The percentages of STAT3 and STAT5B mutations in NKTCL $(n=51), \gamma \delta$-PTCL $(n=24)$ or EATL type II cases $(n=19)$ are shown with piecharts. Apart from the STAT5B Y665F mutation identified using WES, all SNVs identified in this study have been cross-validated on genomic DNA with Sanger sequencing using both forward and reverse primers. *: two NKTCL tumour samples with WTS data was re-classified later as PC- $\gamma \delta$-PTCL due to $\gamma \delta$-TCR expression. One of these two reclassified cases is the sample with the STAT3-G618R mutation. 


\section{Results}

To identify driver mutations of NKTCLs, whole-transcriptome sequencing (WTS), exome sequencing or targeted Sanger sequencing was applied on 53 NKTCL cases (Fig. 1a). First, we validated the mutations detected from our WTS data that may be functionally significant including the mutations in FAS, TP53, $B R A F, M A P 2 K 1, C R E B B P, E P 300$ and MLL2 genes, by Sanger sequencing on the corresponding genomic DNA (Supplementary Table 1). Of note, FAS and TP53 mutations were identified in NKTCLs by traditional Sanger sequencing in previous studies ${ }^{3,4}$. WTS on 17 cases revealed one STAT3 missense single-nucleotide variant $(\mathrm{SNV})(\mathrm{S} 614 \mathrm{R}, \mathrm{G} 618 \mathrm{R}$ and $\mathrm{A} 702 \mathrm{~T})$ in each of three cases (3 of 17, 18\%) (Fig. 1b). A STAT5B missense mutation $(\mathrm{N} 642 \mathrm{H})$ was present in 1 of the $17(6 \%)$ cases (Fig. 1c) and a STAT5B $\mathrm{Y} 665 \mathrm{~F}$ mutation was identified in a WES analysis on a separate paired NKTCL/normal case. Interestingly, all of the STAT3 and $S T A T 5 B$ SNVs were located in the $\mathrm{SH} 2$ domain, a domain critical for STAT activation ${ }^{5}$.

Because all observed SNVs were located in the $\mathrm{SH} 2$ domain, we sequenced the SH2 domains of STAT3 and STAT5B in additional cases. Sanger sequencing of 35 additional NKTCL cases showed a STAT3 D661Y mutation and two STAT5B mutations (Y665F and $\mathrm{N} 642 \mathrm{H}$ ) in three of these 35 screened cases (Fig. 1b,c). These analyses yielded a STAT3 and STAT5B mutation frequency of $5.9 \%$ in all NKTCL patients screened by WTS, WES and/or Sanger sequencing (Fig. 1d, left).

RNA-Seq analysis on three NK cell lines revealed an activating mutation, Y640F, in the SH2 domain of STAT3 in NKYS cells, which was validated by Sanger sequencing (Fig. 1b). Interestingly, two out of three additional NK cell lines (SNK6 and YT) showed STAT3 mutations when screened by Sanger sequencing, raising the STAT3 mutation frequency to $50 \%$ of the six NK cell lines studied (Fig. 1b). However, Sanger sequencing of the SH2 domain (ex14-ex18) of STAT5B in these NK cell lines (Supplementary Fig. 1) did not reveal any $S T A T 5 B$ mutations.

Two recent studies reported the presence of JAK3 A572V, A573V or V722I mutations in NKTCLs 6 .7. However, neither WTS nor hotspot sequencing of 40 NKTCL cases revealed these SNVs, consistent with a recent report ${ }^{8}$. The discrepancy may be due to a variety of factors including the genetic composition of the populations and different stimuli that initiate and sustain the initial phase of NK cell proliferation.

Our gene expression profiling studies found that NKTCL and $\gamma \delta$-PTCL cases share a very similar profile with each other and with normal NK cells ${ }^{9}$, suggesting that these two diseases share many functional pathways and may use similar oncogenic mechanisms. Thus, we screened the $\mathrm{SH} 2$ domains of STAT3 and STAT5B in $3 \gamma \delta$-T cell lines and $24 \gamma \delta$-PTCL cases (15 primary cutaneous (PC)- $\gamma \delta$-PTCL and 9 hepatosplenic (HS) $-\gamma \delta$ PTCL cases). Among the three $\gamma \delta$-T cell lines, we observed a STAT3 Y640F mutation (SNT15) and a STAT3 D661Y mutation (SNT-8), but no STAT5B mutation was observed (Fig. 1b). We identified only a STAT3 Y640F mutation in 1 of the $24 \gamma \delta$-PTCL cases (Fig. 1b). On the other hand, 8 of 24 (33.3\%) cases (four PC and four HS) showed activating STAT5B mutations in the SH2 domain (Fig. 1c,d).

Enteropathy associated T-cell lymphoma (EATL) is an aggressive PTCL with higher incidence in the Europe and $\mathrm{USA}^{10}$. The disease is now classified into two subtypes: type $\mathrm{I}$ is the classical type with TCR- $\alpha \beta$ and is associated with celiac disease and gluten-sensitive enteropathy. EATL type II cases have been shown to frequently express TCR $-\gamma \delta(\sim 78 \%$ in one study $\left.{ }^{11}\right)$, and they are not associated with gluten-sensitive enteropathy. We examined the possibility that STAT3/STAT5B $\mathrm{SH} 2$ domain mutations may play a role in EATL type II pathogenesis and identified STAT5B N642H mutations in 7 of 19 cases (36.8\%) (Fig. 1c,d). Intriguingly, all mutated EATL type II cases have $\gamma \delta$-T-cell receptor expression (7/16 of TCR- $\gamma \delta$-positive cases) underscoring the significance of STAT5B mutations in the neoplastic transformation of $\gamma \delta$-T cells giving rise to different subtypes of $\gamma \delta$-PTCL.

To address whether STAT3 mutations activate the STAT3 pathway, we performed western blotting on six NK cell lines using STAT3 and phospho-STAT3 (Tyr705) antibodies. High pY705-STAT3 expression was seen only in mutant cell lines (Fig. 2a), suggesting that STAT3 is persistently active in the presence of activating mutations in the $\mathrm{SH} 2$ domain. Next, we transduced the NKYS and YT cell lines, which have the activating STAT3-Y640F mutation, with empty vector (EV) or STAT3 shRNA with confirmed activity (Supplementary Fig. 2a,b) to evaluate whether STAT3 silencing inhibits the growth of NK cells. We quantified the percentage of GFP positivity of STAT3 shRNA transduced cells at regular time intervals starting 3 days post transduction, and observed a markedly reduced percentage of $\mathrm{GFP}^{+}$cells compared with vector-only transduced cells, suggesting a strong negative selection pressure after STAT3 knock-down (Fig. 2b-e). We did not observe a decrease in the percentage of $\mathrm{GFP}^{+}$population in STAT3 shRNA transduced KAI3 cells, which express wild-type (WT) STAT3 (Fig. 2f,g). STAT3 protein knock-down efficiency was $\sim 72 \%, \sim 78 \%$ and

\section{Figure 2 | NK cell lines with STAT3 mutations show high pY-STAT3 expression and shRNA-mediated knock-down of STAT3 inhibits NK-cell line} growth. (a) Western blotting was performed on whole-cell lysates (WCL) from six NK cell lines using antibodies to phosphotyrosine (Y705)-STAT3 and STAT3. DHL16 cells, which lack STAT3 activation, were used as a negative control. $\alpha$-Tubulin was used as a control to ensure equal loading of the samples. The names of the cell lines used are indicated above the gel image. The type of the corresponding STAT3 mutation is indicated above the name of the cell lines. ( - ): No STAT3 mutation; N.D., not determined. (b) Representative flow cytometric plots showing the percentage of GFP ${ }^{+}$cells 3 days (day 0 ) and 12 days (day 9) post transduction of NKYS cells with EV or STAT3 shRNA after removing IL2 from the culture medium at day 0. (c) Quantification of the percentage of GFP ${ }^{+}$cells post transduction of NKYS cells with EV or STAT3 shRNA at regular time intervals. In one experiment, cells were cultured in the presence of IL2 $\left(5-7 \mathrm{ng} \mathrm{ml}^{-1}\right.$ ) until 3 days (day 0 ) post transduction and then IL2 was removed from the culture medium. In the second experiment, IL2 was always included post transduction of NKYS cells. Each data point is representative of two biological replicates. (d) Representative FACS plots showing the percentage of GFP ${ }^{+}$cells of YT cell line transduced with EV or STAT3 shRNA 3 days (day 0) or 15 days (day 12) post transduction. (e) Quantification of the percentage of GFP + cells by FACS between 3 and 15 days post transduction with EV or STAT3 shRNA For 1 week before transduction, YT cells were cultured in the presence of IL2. Three days post transduction (day 0), cells were transferred to medium lacking IL2. *: $P<0.05$, Student's t-test. (f) Representative FACS plots showing the percentage of GFP ${ }^{+}$cells in EV or STAT3 shRNA transduced KAI3 cells. (g) Quantification of the percentage of GFP ${ }^{+}$cells after transduction of KAI3 cells with EV or STAT3 shRNA. Three days post transduction (day 0), the percentage of $\mathrm{GFP}^{+}$cells was determined by flow cytometry, and cells were switched to NK culture medium with reduced IL2 (25IU ml ${ }^{-1}$ ). Data represent means \pm s.d. of two biological replicates for panels c,e and $\mathbf{g}$. (h) Western blot images of STAT3 knock-down levels are shown for NKYS,YT and KAI3 cell lines on STAT3 shRNA transduced, GFP ${ }^{+}$sorted cells post transduction with the EV (PLVTH) or STAT3 shRNA (S3S). (i) Quantification of the STAT3 protein knock-down levels after normalization to $\alpha$-Tubulin using the ImageJ program (http://rsb.info.nih.gov/ij/). 
$\sim 39 \%$ in NKYS, KAI3 and YT cell lines, respectively (Fig. 2h,i), which may account for the moderate decrease in growth observed in YT cells after STAT3 knock-down.

Some of the STAT3/STAT5B mutations have been reported in other tumours and are likely oncogenic, but other mutations have not been previously identified. We used several approaches to confirm that these mutations are functionally significant and not passenger mutations or uncommon SNPs. We transduced
KAI3 cells, which lack STAT3 or STAT5B mutations, with each of the STAT3 and STAT5B mutants and determined the \% of $\mathrm{GFP}^{+}$cells post transduction in regular time intervals. With only two exceptions, all STAT5B- or STAT3-mutant transduced KAI3 cells showed significant progressive positive selection under limiting IL2 concentrations compared with WT transduced cells (Fig. 3a,b). STAT3 A702T mutant showed only modest positive selection when compared with WT transduced cells. a

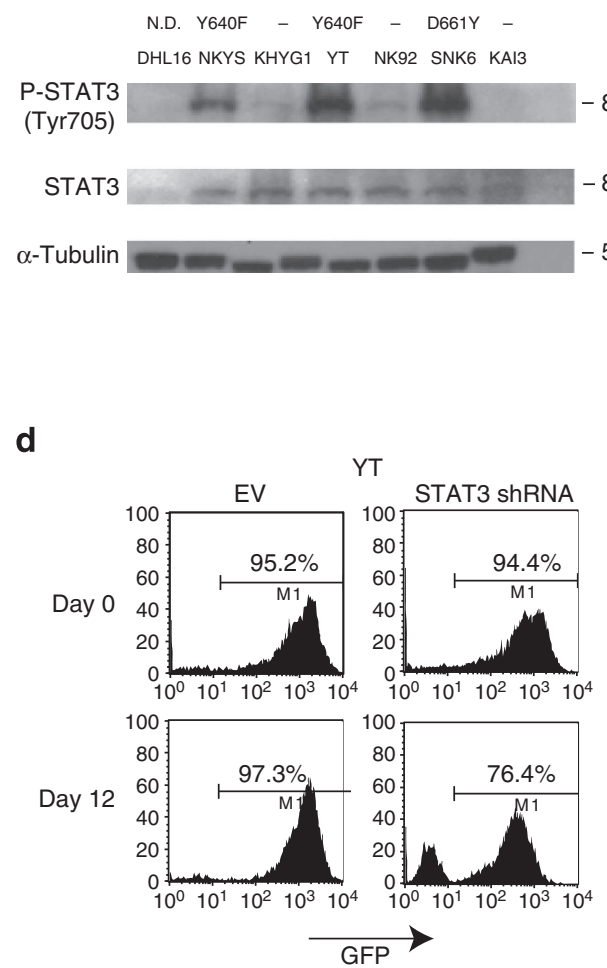

b

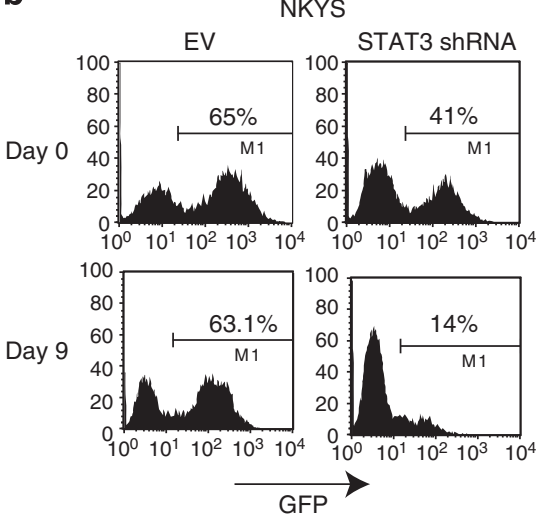

c

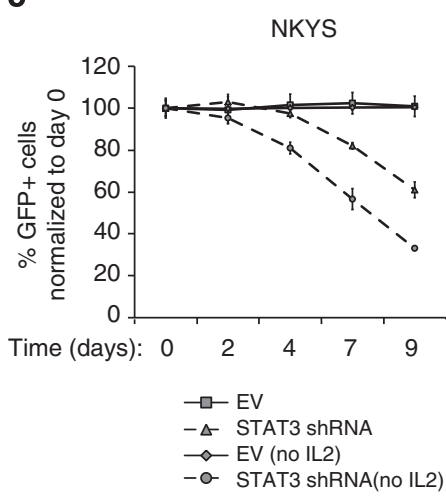

e

YT

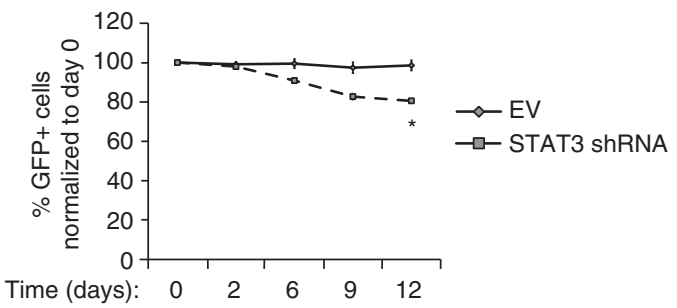

$\mathbf{f}$

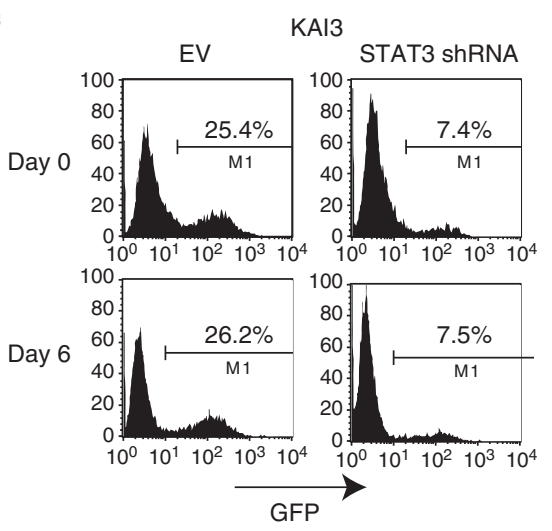

g

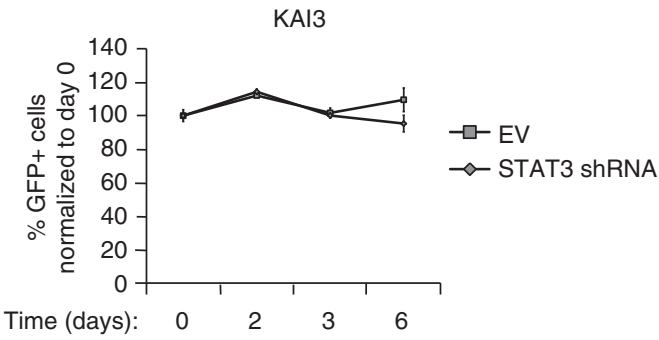

h
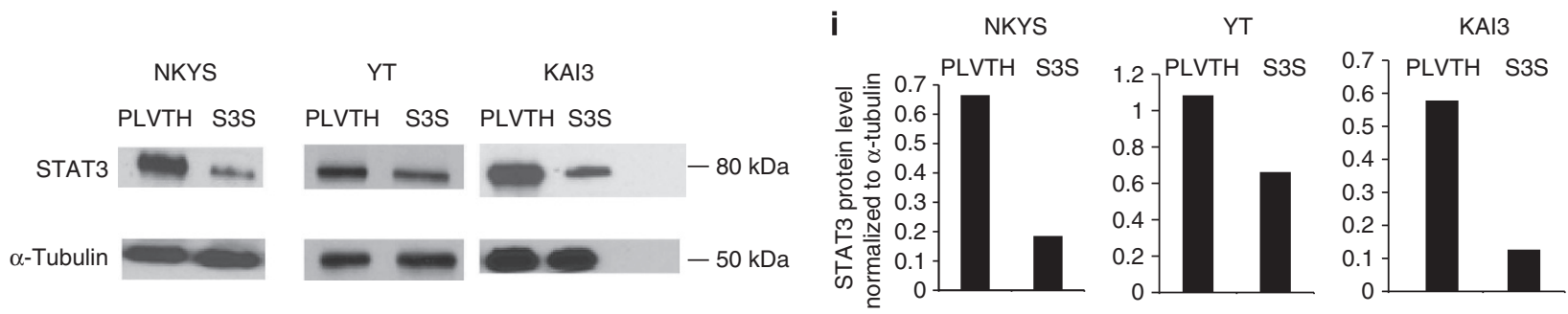
Western blot analysis in cell lines clearly demonstrated an association of STAT3 mutations with increased phosphorylation. As there are no cell lines with STAT5B mutations, we measured the phosphorylation of STAT5B proteins in STAT5B-mutant transduced KAI3 cells and found it to be clearly increased compared with WT transduced cells (Fig. 3c). Next, we transduced STAT5B mutants $(\mathrm{N} 642 \mathrm{H}$ and $\mathrm{I704L})$ into primary human NK cells obtained through coculture with engineered K562 cells, K562-C19-mb21, and observed robust promotion of cell growth in mutant compared with WT transduced cells (Fig. 4). Intriguingly, the effect of STAT5B N642H was prominent and the strongest among all mutants tested in KAI3 cells or normal NK cells.

Next, we evaluated the expression of known STAT5B targets, $I L 2 R \alpha$ (ref. 12), BCL-XL $L^{13}, B C L 2$ (ref. 14), MIR155HG (ref. 15) and HIF2 $\alpha$ (ref. 16) in KAI3 cells transduced with STAT5B mutants $(\mathrm{N} 642 \mathrm{H}$ and $\mathrm{I704L})$ and observed significantly higher expression compared with WT or EV transduced cells (Fig. 5a-e), indicating that the mutants are functionally active and upregulate oncogenic STAT5B targets, which in part accounts for better promotion of growth in NK cells. Next, we performed ChIP-qPCR on STAT5 binding sites for these genes. Compared with KAI3 cells transduced with EV or WT STAT5B, a robust increase in occupancy of STAT5 binding sites were observed in STAT5B-N642H transduced KAI3 cells (Fig. 5f-j), indicating

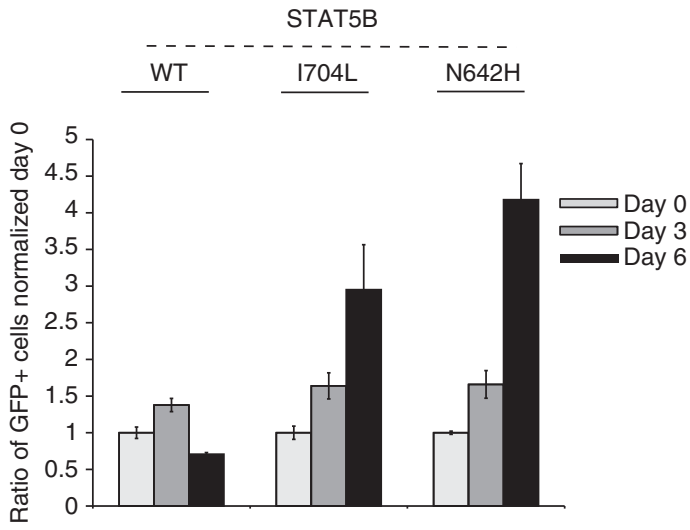

Figure 4 | Ectopic expression of STAT5B 1704L or N642H mutants promotes growth of primary human NK cells. Quantification of the percentage of GFP ${ }^{+}$cells post transduction of primary NK cells with STAT5B-WT or each of two STAT5B mutants (I704L or N642H) at 3-day intervals. The percentage of $\mathrm{GFP}^{+}$cells for each sample is normalized to day 0 . Day 0 indicates 5 days post transduction. The average $\%$ of GFP + cells at day 0 was $4.6,4.9$ and $4.8 \%$ for STAT5B-WT, N642H or $1704 \mathrm{~L}$ transduced cells, respectively. Data are mean \pm s.d. of two biological replicates. Dead cells were stained with $0.5 \mu \mathrm{M}$ of DAPI (Biolegend, cat. no: 422801) to exclude them from quantification. a

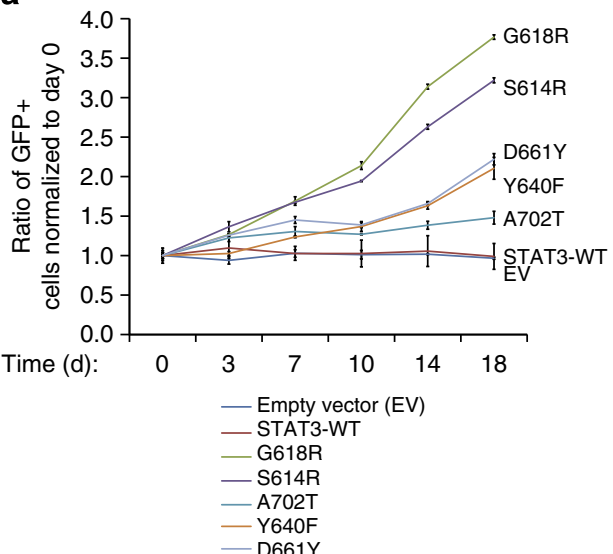

C

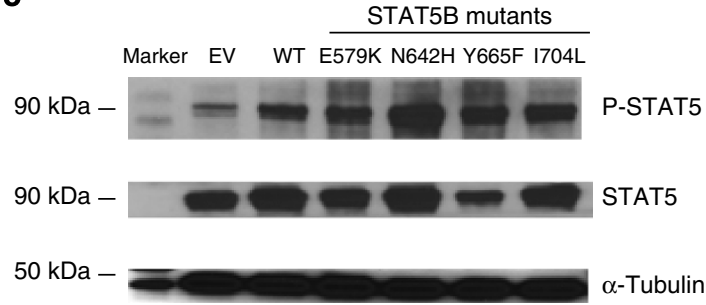

b

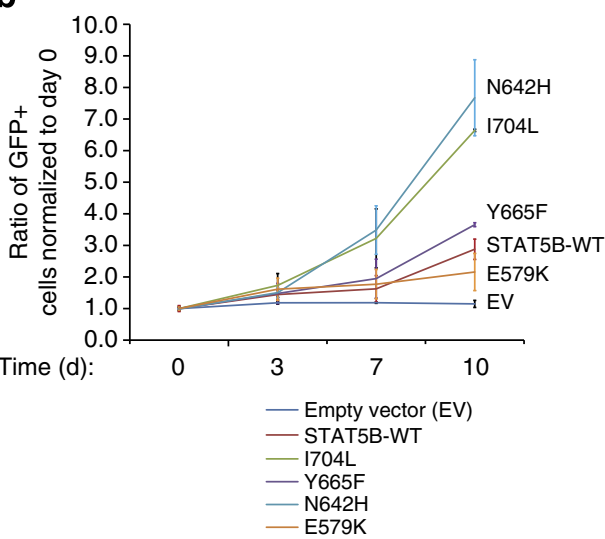

Figure 3 | Ectopic expression of STAT3 or STAT5B mutants promotes growth in KAI3 cells under limiting IL2 concentrations. (a) Quantification of the percentage of $\mathrm{GFP}^{+}$cells post transduction of KAI3 cells with each STAT3 mutant observed in tumour samples. Transduced cells were cultured in regular IL2 concentrations for 4 days, then switched to limiting $\left(25 \mathrm{IU} \mathrm{ml}{ }^{-1}\right) \mathrm{IL} 2$ concentrations. Day $0=4$ days post transduction. Each data point shows the means \pm s.d. of two biological replicates. The average values for the \% of GFP ${ }^{+}$cells at day 0 were 6.9, 3.2, 6.0, 2.8, 4.1, 4.3 and 3.2\% for EV, STAT3-WT, S614R, G618R, Y640F, D661Y or A702T transduced cells, respectively. (b) Quantification of the percentage of GFP+ cells post transduction of KAI3 cells with each STAT5B mutant observed in tumour samples as in $\mathbf{a}$. Means \pm s.d. of two independent experiments with replicates are shown $(n=4)$. The average values for the $\%$ of GFP ${ }^{+}$cells at day 0 were 4.8, 6.8, 5.9, 5.7, 8.2 and 6.7\% for EV, STAT5B-WT, E579K, N642H, Y665F or I704L-mutant transduced cells, respectively. The percentage of GFP ${ }^{+}$cells for each sample is normalized to day 0 in both a,b. (c) P-STAT5(Y699) and STAT5 protein expression levels in KAI3 cells transduced with STAT5B mutants are shown by western blot. Six days post transduction, the GFP + population of STAT5Bmutant transduced cells was isolated by FACS, and then placed into culture medium with limiting $\left(25 \mathrm{IU} \mathrm{ml} \mathrm{I}^{-1}\right)$ concentrations. Whole-cell lysates were collected 11 days post transduction. One experiment representative of three experiments is shown. 
a

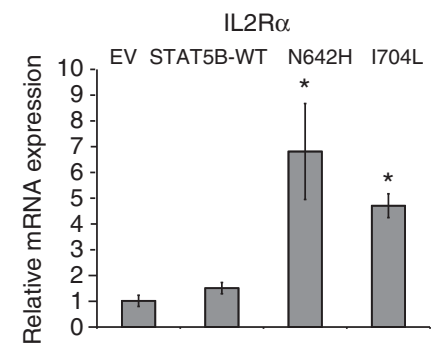

d

MIR155HG

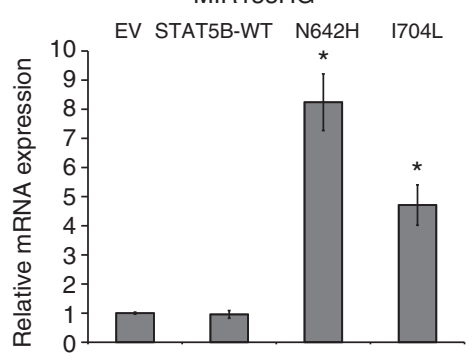

b

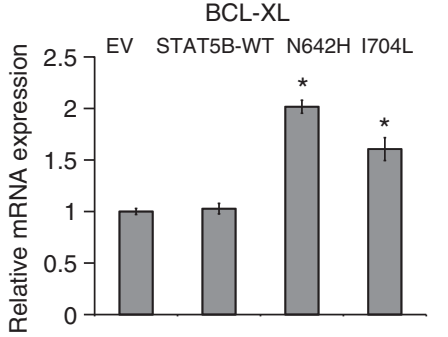

C

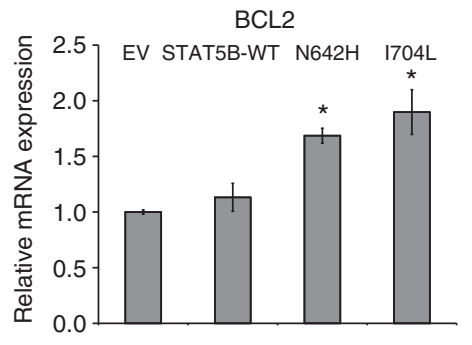

e

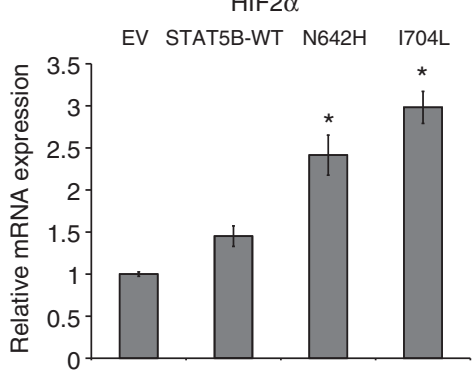

f
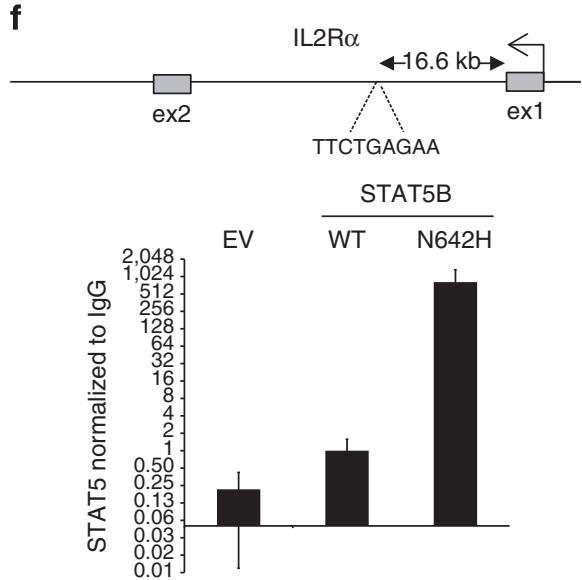

g
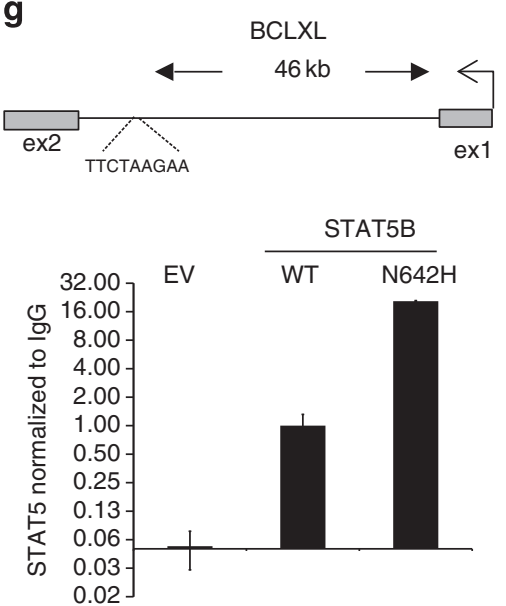

h

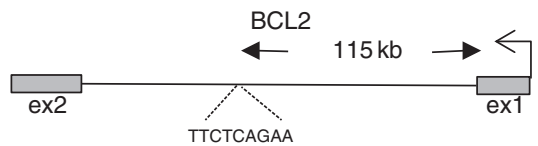

i

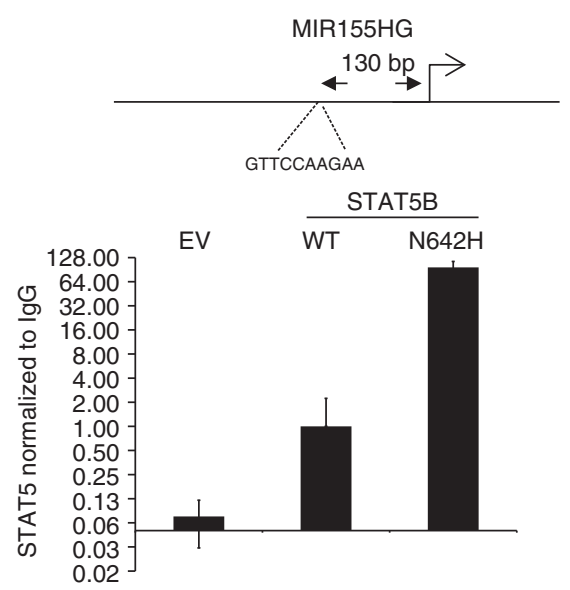

j
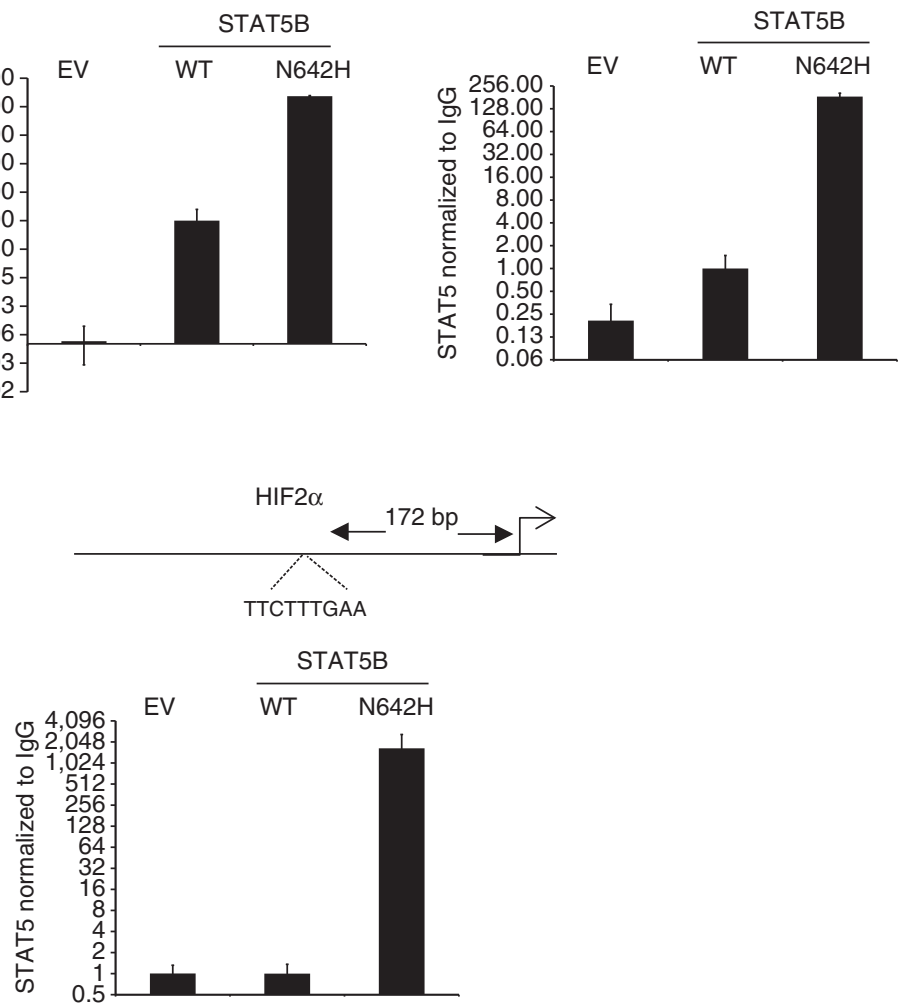

Figure 5 | STAT5B target genes are transcriptionally upregulated in STAT5B-mutant transduced cells through direct binding to conserved STAT5 binding sites. Relative mRNA expression of IL2R $\alpha(\mathbf{a}), B C L-X L(\mathbf{b}), B C L 2(\mathbf{c})$, MIR155HG (d) and, HIF2 $\alpha$ (e) is shown in control vector or STAT5B-mut transduced cells obtained by sorting GFP ${ }^{+}$cells 10 days post transduction, and then culturing for 5 days and 2 days in culture medium with regular or limiting $\left(25 \mathrm{IU} \mathrm{ml}{ }^{-1}\right)$ IL2 concentrations, respectively. Means \pm s.d. of two independent experiments (total combined samples $=4$ ) are shown. ${ }^{\star} P<0.01$ compared with WT, Student's t-test. ChIP-q-PCR results for known STAT5 binding sites are shown for IL2R $\alpha(\mathbf{f}), B C L-X L(\mathbf{g}), B C L 2$ (h), MIR155HG (i) and HIF2 $\alpha(\mathbf{j})$ in EV, STAT5B-WT or STAT5B-N642H transduced KAI3 cells. Data are mean \pm s.d. of four replicates. STAT5 pull-down normalized to lgG control as a fold difference compared with STAT5B-WT sample is shown in the $y$-axis using a log scale. STAT5B consensus sites and their approximate distance to the TSS sites are indicated. 
that increased STAT5B binding due to the mutation upregulates the expression of the target genes. Altogether, these results suggest that the STAT3/STAT5B mutations are oncogenic, driver mutations.

STAT5B N642H is the most frequent mutation identified in $\gamma \delta$-PTCLs and appears to have the highest functional potency. Hence, we sought to determine the molecular basis of the functional alterations resulting from this mutation through structural analysis. To mimic homodimerization, we docked a tyrosine-phosphorylated STAT5B peptide into the WT and mutant SH2 domains (Fig. 6a-c). Molecular docking showed that the peptide has a significantly higher binding affinity to the $\mathrm{N} 642 \mathrm{H}$ mutant than the WT (Fig. 6d) largely due to the direct electrostatic interaction of the mutant histidine with the phosphotyrosine. To confirm these modelling studies, we produced non-phosphorylated WT and N642H STAT5B (residues 128 to 717), purified each to homogeneity, and performed SPR studies. The WT and mutant STAT5B proteins were coupled a
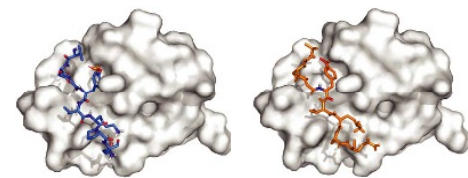

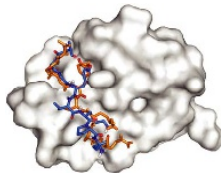

d

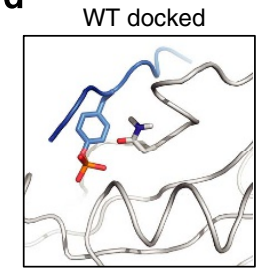

Predicted binding free energy : $-101.6 \mathrm{kj} \mathrm{mol}^{-1}$

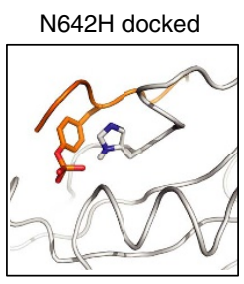

Predicted binding free energy : $-125.3 \mathrm{kj} \mathrm{mol}^{-1}$

e

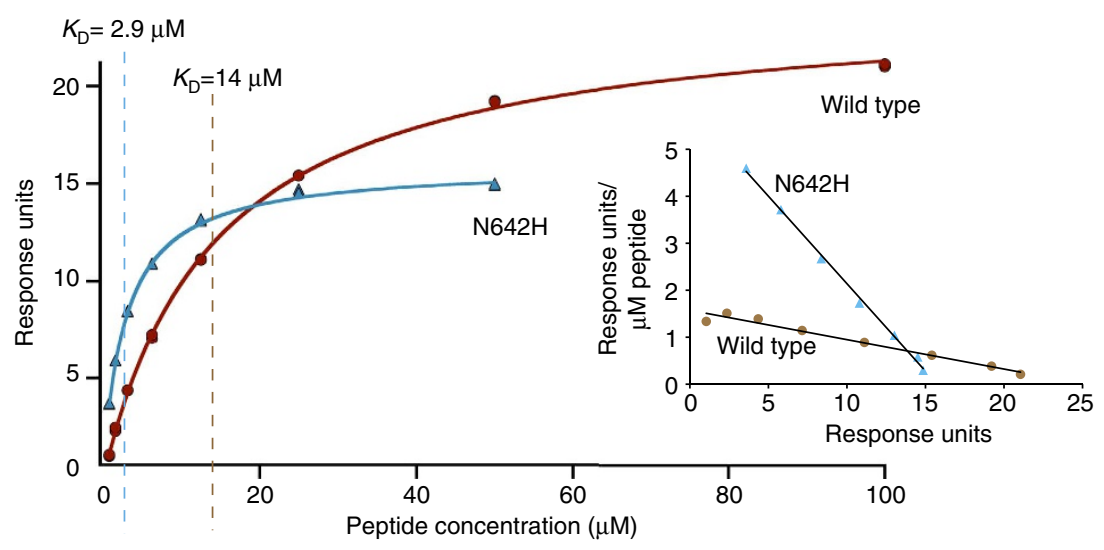

f

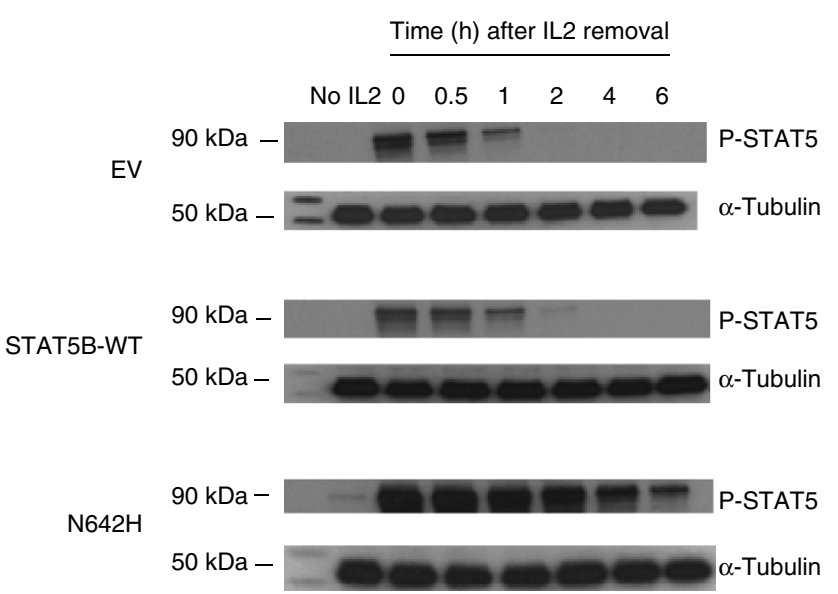

Figure 6 | Three-dimensional modelling and SPR analysis of the $\mathbf{N 6 4 2 H}$ mutant shows stronger affinity for the phosphorylated tyrosine Y699. The phosphorylated STAT5B peptide involved in homodimerization was docked into the SH2 domain of either WT (a) or N642H (b) STAT5B. The overlay of these structures (c) shows little difference in the docking poses. In both cases, the N642 or H642 residue was found to be in close proximity to the phosphorylated tyrosine on the STAT5B peptide (d). The binding energy of these docked structures was calculated for both the WT ( $-101.5 \mathrm{~kJ}$ mol ${ }^{-1}$ ) and $\mathrm{N} 642 \mathrm{H}\left(-125.3 \mathrm{~kJ} \mathrm{~mol}^{-1}\right.$ ). (e) SPR binding isotherms of phosphopeptide with STAT5B-WT (brown circles) and N642 mutant (blue triangles) of a representative experiment is shown. Calculated $K_{\mathrm{D}}$ values of 14 and $2.9 \mu \mathrm{M}$ for STAT5B-WT and N642H mutant, respectively, are indicated by vertical lines for the representative experiment. An inset shows the Scatchard plot of the same data. The experiment was repeated for four times, and the means \pm s.d. of $K_{\mathrm{D}}$ values for $\mathrm{WT}$ and $\mathrm{N} 642 \mathrm{H}$ are $15.6 \pm 1.4 \mu \mathrm{M}$ and $3.23 \pm 0.33 \mu \mathrm{M}$, respectively. $P<0.01$ for $\mathrm{N} 642 \mathrm{H}$ compared with WT, Student's $t$-test. (f) Western blot image showing P-STAT5(Y699) and STAT5 levels before or after culturing EV, STAT5B-WT or N642H mutant transduced, GFP-sorted YT cells in the absence (no IL2) or the presence of IL2. YT cells were cultured in the absence of IL2 in each stage of the experiment including 1 week before transduction. The image is representative of two independent western blots. 
to individual channels of a CM5 SPR chip, and the phosphopeptide KAVDG(p)YVKPQI was passed over each channel at increasing concentrations $(0.8$ to $100 \mu \mathrm{M})$ in duplicates. The binding affinity of the peptide to the WT and $\mathrm{N} 642 \mathrm{H}$ mutant was determined by fitting the equilibrium saturation point as a function of peptide concentration. The dissociation constants for the $\mathrm{N} 642 \mathrm{H}$ mutant and WT STAT5B on average were 3.2 and $15.6 \mu \mathrm{M}$, respectively (Fig. 6e). While these measurements represent a $\sim$ fivefold difference for the peptide to the monomeric STAT5B-WT compared with $\mathrm{N} 642 \mathrm{H}$ mutant, it is important to note that phosphoSTAT5B forms a homodimer and thus there are two phosphotyrosine binding sites. Consequently,
$K_{\text {Dimer }}=K$ Monomer1 $\bullet K_{\text {Monomer2 }}$ that translates to a 25 -fold increased association constant for the dimer. Hence, the homodimeric $\mathrm{N} 642 \mathrm{H}$ mutant is substantially more stable than the WT. This finding supports the molecular model and the in vitro cell based assay observations.

To address whether the STAT5B-N642H mutation is associated with prolonged STAT5B activation in the presence of acute JAK-STAT5 pathway activation, we performed western blot on IL2-activated YT cells in a time-course experiment to evaluate P-STAT5 expression. The JAK-STAT5 pathway is known to be inducible in this cell line ${ }^{17}$. We incubated EV, STAT5B-WT or $\mathrm{N} 642 \mathrm{H}$-mutant transduced YT cells with IL2 for $30 \mathrm{~min}$, and a

STAT3 $72 h$

EV STAT3-WT G618R Y640F

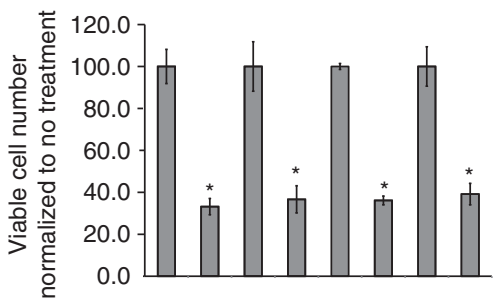

b

ATSB-WT N642H 1704L

AZD1480 (0.5 uM): -+-+-+-+

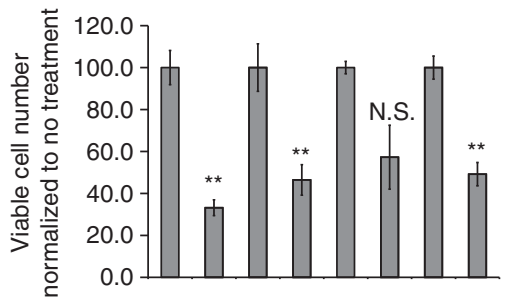

C
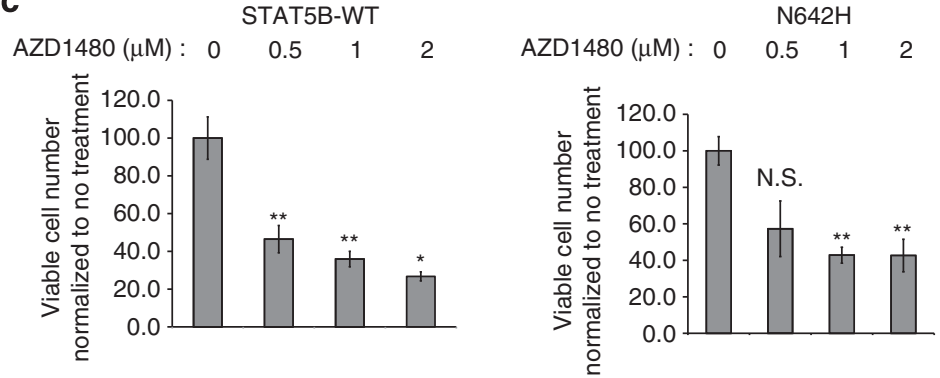

d
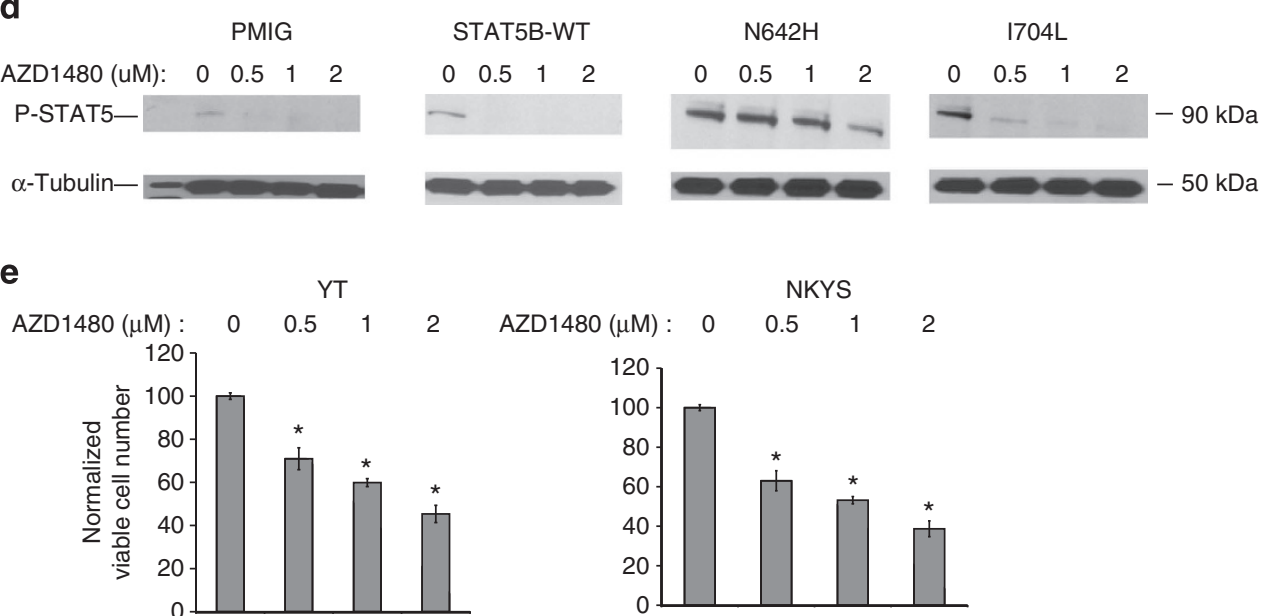

Figure 7 | AZD1480 inhibits STAT3/STAT5B-mutant transduced NK cells. STAT3-mutant (a) or STAT5B mutant (b) transduced, GFP ${ }^{+}$-sorted KAI3 cells were treated with $0.5 \mu \mathrm{M}$ AZD1480 for $72 \mathrm{~h}$. Viable cell number was determined using Vi-cell XR cell viability analyser (Beckman Coulter Inc.) as described in Supplementary Methods and normalized to the corresponding untreated sample. Means \pm s.d. of two biological replicates are shown. (c) Normalized viable cell number of STAT5B-WT (left) or N642H-mutant (right) transduced KAI3 cells treated with progressively increasing doses of AZD1480 for $72 \mathrm{~h}$. Each column represents mean \pm s.d. of three biological replicates. (d) P-STAT5 (Y699) levels were analysed by western blot on EV, STAT5B-WT, N642H or 1704L transduced KAI3 cells treated with progressively increasing doses $(0.5 \mu \mathrm{M}, 1$ or $2 \mu \mathrm{M})$ of AZD1480 for $4 \mathrm{~h}$ (e) Normalized numbers of viable STAT3-mutated NKYS and YT cells treated as in (c) are shown ${ }^{\star} P<0.01$ versus no treatment; ${ }^{\star} P<0.05$ versus no treatment; Student's t-test. NS: not significant. 
evaluated P-STAT5 expression up to $6 \mathrm{~h}$ after IL2 withdrawal. P-STAT5 expression disappeared $1 \mathrm{~h}$ after transient IL2 stimulation in EV or STAT5B-WT transduced cells whereas it persisted for $>6 \mathrm{~h}$ in $\mathrm{N} 642 \mathrm{H}$ transduced cells (Fig. 6f). These results suggest that the $\mathrm{N} 642 \mathrm{H}$ mutation increases affinity of phospho-STAT5B dimerization and thereby resulting in far more persistent activation.

We next tested whether a JAK1/2 inhibitor can inhibit cell growth of STAT5B or STAT3-mutant transduced KAI3 cells. Most NKCL and $\gamma \delta$-PTCL cell lines are still dependent on IL2 which signals mainly through JAK1/2 to activate STAT3 and STAT5 (refs 18,19). JAK inhibitors are expected to be effective in interrupting this signalling pathway in cells with WT STAT3 and STAT5. However, it is unclear whether the inhibitor would effectively inhibit this pathway at a tolerable dosage in cells harbouring the mutants. We treated STAT3 or STAT5B mutant and WT transduced KAI3 cells with $0.5 \mu \mathrm{M}$ AZD1480, a selective JAK1/2 inhibitor ${ }^{20}$. We quantified viable cells at $72 \mathrm{~h}$ post treatment and observed inhibition of growth in all the mutant STAT3 and STAT5B transduced cells (Fig. 7a,b). With higher concentrations of AZD1480, we observed up to 60\% inhibition in STAT5B N642H-mutant transduced cells (Fig. 7c, right), associated with reduced STAT5 phosphorylation in a dosedependent manner (Fig. 7d). Next, we treated YT and NKYS cells, which have STAT3 mutations, with the JAK1/2 inhibitor and observed dose-dependent inhibition of cell growth (Fig. 7e). These data suggest that JAK1/2 inhibitors may have therapeutic efficacy for NKTCL and $\gamma \delta$-PTCL patients with the mutations. This may have immediate clinical implication as JAK1/2 inhibitors are already approved for myeloproliferative disorders $^{21,22}$ so they are available for trials in NKTCL or $\gamma \delta$ PTCL patients while STAT3 and STAT5B inhibitors are still not clinically available. Further development of small-molecule inhibitors targeting STAT3 or STAT5B dimerization or DNA binding may synergize with JAK1/2 inhibitors to improve the outcome in these diseases with currently dismal prognosis.

Although STAT3 or STAT5B mutations have been reported in malignancies such as B-cell lymphomas ${ }^{23}$, angioimmunoblastic T-cell lymphoma ${ }^{24}, \mathrm{CD} 0^{+}$T-cell lymphoma ${ }^{25}$, the indolent LGLL diseases $26-28$ and recently in T-cell prolymphocytic leukaemia ${ }^{29}$, which also included functional characterization of STAT5 mutations, our study describes prevalent activating STAT5B and STAT3 mutations in aggressive lymphomas of NK and $\gamma \delta$-T cell of origin. Importantly, we provided strong in vitro functional data that support an oncogenic role of these mutants as well as mechanistic insight into how the $\mathrm{N} 642 \mathrm{H}$ mutant acquires enhanced functional activities.

The frequency of activating STAT3 mutations was much higher in NK (50\%) and $\gamma \delta$-T cell lines (67\%) compared with NKTCL $(5.9 \%)$ or $\gamma \delta$-PTCL patient samples $(8.3 \%)$. This suggests that JAK-STAT3 pathway activation may be more critical in cell survival independent of stromal components and that consequently the activating mutations are selectively enriched in cell lines. Intriguingly, STAT5B mutations were far more frequent in $\gamma \delta$-T-cell lymphomas including multiple subtypes: HS, EATL type II and other mucocutaneous $\gamma \delta$-PTCL (34.9\%) than in NKTCL cases $(5.9 \%)$ suggesting a pivotal role of STAT5B in $\gamma \delta$-PTCL pathogenesis.

\footnotetext{
Methods

Patient samples and cell lines. The phenotypic characteristics of all NKTCL, $\delta \gamma$ PTCL and EATL type II cases $(n=94), \mathrm{NK}$ and $\delta \gamma$-T cell lines $(n=9)$ used in this study are summarized in Supplementary Table 2 . Informed consent was obtained from all patients in accordance with the Declaration of Helsinki, and use of patient materials and information was approved by the institutional review boards of the UNMC, West China Hospital Sichuan University, and the participating institutions
} of the Tenomic Consortium. KHYG1, KAI3 cell lines were obtained from the
Health Science Research Resource (Osaka, Japan). YT and NK-92 cell lines were provided by the German Collection of Microorganism and Cell Culture (GCMCC) (DSMZ, Braunschweig, Germany). Two NK cell lines (SNK6, NKYS) and the $\gamma \delta$-T cell lines (SNT8, SNT13, and SNT15) were obtained from Dr Norio Shimizu (Tokyo Medical and Dental University). Culture conditions of NK and $\gamma \delta$-T cell lines were as described previously ${ }^{30,31}$. The DHL16 cell line, purchased from ATCC (American Type Culture Condition), was cultured in RPMI-1640 (GibcoInvitrogen) including $10 \% \mathrm{FBS}$; penicillin $\mathrm{G}\left(100\right.$ units $\left.^{-1}\right)$ and streptomycin $\left(100 \mu \mathrm{g} \mathrm{ml}^{-1}\right)$ at $37^{\circ} \mathrm{C}$ in $5 \% \mathrm{CO}_{2}$.

WTS and data analysis. RNA sequencing was performed on resting NK cells, NK cells activated by IL2 or by K562-Clone9-mb21, 17 NKTCL cases and 3 NK cell lines. Briefly, 100-bp paired-end libraries were prepared with the TrueSeq RNA preparation kit (Illumina Inc., San Diego, CA), and high-throughput sequencing was performed at the UNMC Next Generation Sequencing Core facility and Tufts University (TUCF) Genomics Core Facility using Illumina Genome Analyzer IIX or HiSeq 2000 Sequencing systems. FASTQC reports were evaluated for each sample to evaluate the quality of basic statistics. Two different pipelines were used to generate the SNVs. The main pipeline used for SNV detection was described previously $^{32}$ with the following addition. In addition to the NCBI SNP database (dbSNP) and 1,000 Genomes project ${ }^{33}$, three normal NK samples were used to filter out the SNPs. The presence of the SNVs was evaluated by visualizing the SNVs using Integrative Genomics Viewer software (IGV) (http:// www.broadinstitute.org/igv). Finally, the Cosmic release v69 (http:// cancer.sanger.ac.uk/cancergenome/projects/cosmic/) was used to annotate the variants observed in previous studies. The secondary pipeline used for SNV detection is as follows: The reads were aligned to the human reference genome (NCBI GRCh37) using the BWA aligner ${ }^{34}$ with paired-end (sampe) mode and with default options. After merging BAM files, PCR duplications were also removed. Then, GATK tool ${ }^{35}$ was used to realign indel-containing reads to the reference genome. After realignment, GATK UnifiedGenotyper was used to generate SNP and indel callsets for 24 (21 malignant and three normal NK samples) RNA-Seq samples, using a merged BAM file including all 24 data sets with specific IDs. Variant Quality Score Recalibration filter was applied using the GATK resource bundle 1.2 to help minimize false positives. Then, ANNOVAR tool ${ }^{36}$, version 201302-11, was used to annotate the detected SNPs and indels. For gene and filter annotation, the April 2012 version of the annotation database (hg191000g2012apr) and dbSNP version 137 was used. For comparison against the 1,000 Genomes Project, the data 1000g2012apr was used. Lastly, the SNVs present in three normal NK samples were filtered out. The basic statistics of RNA-Seq are shown in Supplementary Table 3. Furthermore, the number of SNVs and their annotations identified by the primary RNA-Seq pipeline for 17 NKTCL cases are shown in Supplementary Fig. 3 and Supplementary Table 4, respectively.

Whole genome amplification. Whole genome amplification (WGA) of the NKTCL $(n=20)$ or $\gamma \delta$-PTCL $(n=4)$ cases and KAI3 cell line was performed using the Repli-g kit (Qiagen Inc., Valencia, CA). $50 \mathrm{ng}$ of tissue material was used as a template for amplification. The sensitivity of mutation detection was evaluated by applying Sanger sequencing on the G to A mutation detected in the intron4/exon5 splice junction of $P R D M 1$ detected previously (Supplementary Fig. 4A) ${ }^{30}$. Uniform linear amplification of genomic DNA from each NK sample was tested with PCR, which generated $\sim 3 \mathrm{~kb}$ amplicons using KAI3 cell line or NKTCL cases (Supplementary Fig. 4B,C). In addition, WGA DNA from NKTCL cases was run on TAE-agarose gels, which showed that WGA DNA contains large fragments $(>10 \mathrm{~kb}$ ) (Supplementary Fig. 4D).

Mutation validation by Sanger sequencing. Sanger sequencing was performed on DNA from cryopreserved or FFPE tissues, or CDNA if only RNA was available. Sequencing was focused on the SH2 domain of STAT3 and STAT5B and the previously reported mutation hotspots for JAK3. The genomic DNA sequences around the JAK3 A572V, A573V and V722I SNVs was obtained using UCSC (http://genome.ucsc.edu/) genome browser. PCR primers covering SNVs were designed with the PrimerQuest software (IDT DNA technologies, Coralville, IA). The primers were optimized with gradient PCR, and the forward and reverse primers used for PCR amplification of WGA or FFPE gDNA or cDNA samples were used for Sanger sequencing. Analysis of the sequences was performed using Vector NTI 10.3.0 (Invitrogen, Carlsbad, CA) and Sequence Scanner Software v1.0 (Applied Biosystems Inc.).The primers used for Sanger sequencing are shown in Supplementary Table 5.

NK cell isolation and activation for RNA-Seq. Primary human NK cells were isolated from peripheral blood lymphocytes using a human NK cell isolation kit (Miltenyi Biotec, Auburn, CA) as described previously ${ }^{37}$. The purity of NK cells was evaluated by CD56-APC and CD3-PE double staining, and samples with $>95 \% \mathrm{CD}^{2} 6^{+} \mathrm{CD}^{-}$cells were used for RNA-Seq. Resting NK cells were cultured in the presence of $100 \mathrm{IU}$ of IL2 for $48 \mathrm{~h}$ to obtain activated NK cells. Higher levels of NK cell activation were achieved by coculturing freshly isolated peripheral blood lymphocytes with engineered K562 cells, K562-Clone9-mb21, as described in detail before ${ }^{37,38}$. 
Quantitative reverse transcription PCR (q-RT-PCR). mRNA expression was determined with q-RT-PCR. RNA isolation and reverse transcription were performed as described previously 37,39 . Real-time PCR was performed using DyNAmo HS SYBR Green qPCR Kit (Thermo Scientific Inc.) with CFX Connect (Bio-Rad, Hercules, CA) real-time thermocycler. Melting curve analysis was performed to ensure amplification specificity. The $\Delta \Delta \mathrm{Ct}$ method was used to calculate the relative mRNA expression level. RPL13A was used for normalization of gene expression. Q-RT-PCR primers used in this study are as follows: $B C L-X L$ forward: $5^{\prime}$-CTTACCTGAATGACCACCTAGAG- $3^{\prime}$; BCL-XL reverse: $5^{\prime}$ - AGGAACCAG CGGTTGAAG-3'; IL2R $\alpha$ forward: 5'-AGCGAGCGCTACCCACTTCTAAAT-3'; IL2R $\alpha$ reverse: 5'-AGGGTGGAGAGAGTTCCATACCAT-3'; BCL2 forward: $5^{\prime}$ - CATGTGTGTGGAGAGCGT CAA-3'; BCL2 reverse: $5^{\prime}$-CAGAGACAGCCA GGAGAAATCA-3. MIR155HG forward: 5'-ACGGTTGTGCGAGCAGAGAAT CTA - $3^{\prime}$; MIR155HG reverse: $5^{\prime}$-CTCATCTAAGCCTCACAACAACCT-3'; HIF2 $\alpha$ forward: $5^{\prime}$-GTGCTCCCACGGCCTGTA- ${ }^{\prime}$; HIF $2 \alpha$ reverse: $5^{\prime}$-TTGTCACACC TATGGCATATCACA- $3^{\prime}$.

Western blot. Western blot was performed as described previously with the following modifications ${ }^{30}$. RIPA buffer supplemented with a protease inhibitor cocktail (Sigma-Aldrich, St Louis, MO) and phosphatase inhibitor cocktails 2 and 3 (Sigma-Aldrich) was used to prepare the whole-cell lysate. Twenty micrograms protein/sample was used for western blot. BSA (Sigma-Aldrich) was used instead of non-fat dry milk during blocking. The primary antibodies used for western blotting are as follows: STAT3 (Cell Signaling Inc., Danvers, MA), STAT5 (3H7) Rabbit mAb \#9358 (Cell Signaling), phospho-STAT5 D47E7 Rabbit mAb \# 4322 (Cell Signaling), phospho-STAT3 (Cell Signaling) and $\alpha$-Tubulin (Sigma-Aldrich). The working dilution of $\alpha$-Tubulin is 1:50,000. The working dilution for all other antibodies is 1:1,000. Uncropped representative WB images are shown in Supplementary Fig. 5.

STAT3 shRNA expression in NK cell lines. The lentiviral construct used for STAT3 knock-down was described previously ${ }^{40}$. Lentiviral transduction of NK cell lines or DHL16 was performed following the protocol used for retroviral transduction $^{37}$ with the following modifications: $4 \mu \mathrm{g}$ PLVTH or PLVTH-S3S was cotransfected with $2 \mu \mathrm{g}$ of PMD2G and $2 \mu \mathrm{g}$ psPAX2 packaging constructs into the $293 \mathrm{~T}$ cell line to generate lentiviral particles. Transduction was performed once rather than twice. Transduction efficiency was determined with fluorescenceactivated cell sorting (FACS) 3 days post transduction.

Generation and expression of the STAT3 or STAT5B constructs. WT STAT5B was PCR cloned with the high-fidelity PfuUltra II Fusion HS DNA Polymerase (Agilent Technologies, Palo Alto, CA) using NK92 cell line cDNA as the template and then cloned into the multiple cloning site of the PMIG expression vector using NotI and SalI restriction sites. Similarly, WT STAT3 was PCR cloned into pMIG from KAI3 cell line cDNA using NotI and SalI sites. Diagnostic mapping and full insert sequencing was performed. These WT STAT3 or STAT5B constructs were used as templates for site-directed mutagenesis to generate the STAT3 or STAT5B mutants used for functional studies as described below apart from the STAT3Y640F-pMIG construct, which was PCR cloned with PfuUltra II Fusion HS DNA Polymerase using the cDNA from NKYS cells, which have the STAT3 Y640F mutation.

STAT3 or STAT5B SNVs observed in NKTCL, $\gamma \delta$-PTCL or EATL type II samples (patient samples or cell lines) were generated using the Quick-Change Site-Directed Mutagenesis Kit (Agilent technologies, Santa Clara, CA) according to the manufacturer's instructions using WT STAT3 or STAT5B-pMIG vectors (Supplementary Fig. 6). Diagnostic restriction mapping and full sequencing of the inserts were performed to ensure the presence of introduced mutation and absence of another SNV. The primers used for site-directed mutagenesis (SDM) are as follows: STAT3-S614R-SDM-F: 5'-GAAAGCAGCAAAGAAGGACGCGTCACTT TCACTTGG-3'; STAT3-S614R-SDM-R: 5'-GTGACGCCTCCTTCTTTGCGGCT TTCACTGAATCTT-3' ${ }^{\prime}$; STAT3-G618R-SDM-F: 5'-GAAAGCAGCAAAGAAGG ACGCGTCACTTTCACTTGG-3'; STAT3-G618R-SDM-R: 5'-CCAAGTGAAAG TGACGCGTCCTTCTTTGCTGCTTTC-3'; STAT3- D661Y-SDM-F: 5'-TGGGC TATAAGATCATGTATGCTACCAATATCCTGG-3'; STAT3-D661Y-SDM-R: 5'-CCAGGATATTGGTAGCATACATGATCTTATAGCCCA-3'; STAT3-A702TSDM-F: 5' -AGCTGACCCAGGTAGCACTGCCCCATACCTGAAG-3'; STAT3A702T-SDM-R: 5' -CTTCAGGTATGGGGCAGTGCTACCTGGGTCAGCT-3'; STAT5B-E579K-SDM-F: 5'-GGTTTGACGGTGTGATGAAAGTGTTAAAAAAA CATC-3'; STAT5B-E579K-SDM-R: 5'-GATGTTTTTTTAACACTTTCATCACA CCGTCAAACC-3'; STAT5B-N642H-SDM-F: 5'-GGAAAGAATGTTTTGGCAT CTGATGCCTTTTACC-3'; STAT5B-N642H-SDM-R: 5'-GGTAAAAGGCATCA GATGCCAAAACATTCTTTCC-3'; STAT5B-Y665F-SDM-F: 5'-GCTTGGGAGA CTTGAATTTCCTTATCTACGTGTTTC-3'; ${ }^{\prime}$ STAT5B-Y665F-SDM-R: 5'-GAAA CACGTAGATAAGGAAATTCAAGTCTCCCAAGC-3'; STAT5B-I704L-SDM-F: 5'-GGATACGTGAAGCCACAGCTCAAGCAAGTGGTCCCTG-3'; STAT5B-

I704L-SDM-R: 5' -CAGGGACCACTTGCTTGAGCTGTGGCTTCACGTATCC- ${ }^{\prime}$.

Retroviral transduction of NK cell lines was performed as previously described $^{39}$ with the following modifications: $4 \mu \mathrm{g}$ of pMIG or pMIG vectors expressing WT or mutated STAT3/STAT5B gene was cotransfected with $4 \mu \mathrm{g}$ of the packaging construct PCL-Ampho into the 293T cell line. A single transduction was performed. Transduction efficiency was determined with flow cytometry on $\mathrm{GFP}^{+}$cells 2-4 days post transduction. KAI3 cells were cultured in the presence of $20 \%$ FBS to increase transduction efficiency.

Conditioning primary NK cells for retroviral transduction. Primary human NK cells were expanded using a special ex vivo system that involves coculturing primary human NK cells with an engineered NK cell target, K562-Cl9-mb21, which activates and induces proliferation of NK cells robustly as described before ${ }^{37,39}$. The expansion procedure is described briefly as follows: First, primary human NK cells were isolated by negative selection using EasySepHuman NK cell enrichment kit (Stemcell technologies,Vancouver,Canada). Then, primary NK cells were admixed in a 1:2 ratio with $100 \mathrm{Gr}$ irradiated $\mathrm{K} 562-\mathrm{Cl} 9-\mathrm{mb} 21$ cells, which express CD86, 4-1BBL and mIL21 on their surface, and cultured in NK cell expansion medium ${ }^{38}$. Cells were spun down at $400 \mathrm{~g}$ for $5 \mathrm{~min}$, and the culture medium was renewed every 3 days with fresh culture medium, keeping the cell density at 250,000 cells per $\mathrm{ml}$ after every subculture. Nine days after coculture started, cells were immunostained with CD56-PE (Biolegend, San Diego, CA) and CD3-FITC (Biolegend) antibodies to determine the NK cell purity by FACS. On the same day purity was determined, primary NK cells were transduced with WT or mutant STAT5B retroviral constructs.

Determination of positive/negative selection of transduced cells. STAT3 shRNA or STAT3/STAT5B mutant transduced NK cell lines were tracked by quantification of the $\mathrm{GFP}^{+}$cells using flow cytometry after transduction to determine negative or positive selection of cells, respectively, because GFP was used as the marker of transduction. The following flow cytometers were used for determination of $\mathrm{GFP}^{+}$cells: FACS Calibure (BD Biosciences), BD LSRFortessa (BD Biosciences) and Gallious (Beckman Coulter Inc.) Autofluorescent cells, which emit both green and orange, represent false positive, untransduced cells, were filtered out through proper gating. During quantification of GFP ${ }^{+}$cells in transduced primary NK cells, dead cells were labelled and filtered out by staining the cells with $0.5 \mathrm{ug} \mathrm{ml}^{-1}$ DAPI (Biolegend, cat.no: 422801 ) for $10 \mathrm{~min}$ before flow cytometry.

ChIP-q-PCR. Ten million cells isolated from GFP-sorted, EV, STAT5B-WT or N642H-mutant transduced KAI3 cells were used for chromatin immunoprecipitation using ChIP-IT Express Enzymatic (Active Motif, Carlsbad, CA) following the manufacturer's recommendations. The procedure is described briefly as follows: the enzymatic digestion time was optimized as $10 \mathrm{~min}$ based on the TAE-agarose gel image. Cells $\left(10^{*} 10^{6}\right)$ per sample were fixed with $1 \%$ formaldehyde. After enzymatic fragmentation, STAT5 (3H7) Rabbit mAb \#9358 (Cell Signaling Inc.) and rabbit anti-IgG Control (Abcam Inc., Cambridge, MA) antibodies were used side-by-side for immunoprecipitations. Twenty micrograms of chromatin/reaction was immunoprecipitated using dilutions of STAT5 or IgG antibody based on manufacturers' recommendations. After elution of DNA, reversal of DNA crosslinks, and proteinase $\mathrm{K}$ treatment, q-PCR was performed using $2 \mu \mathrm{l}$ of gDNA in replicate. STAT5 immunoprecipated DNA levels were normalized to the levels of IgG immunoprecipitated DNA for each sample. STAT5 binding sites reported before for $I L 2 R a$ (ref. 12), BCL2 (ref. 14), BCL-XL (ref. 12), HIF2 $\alpha$ (ref. 16) and MIR155HG (ref. 15) were evaluated. The sequences of ChIP-q-PCR primers used in this study is as follows: $I L 2 R \alpha$-ChIP-q-PCR-F: $5^{\prime}$-AAAACCAATTTCTTGGGA TGG-3'; IL2R $\alpha$-ChIP-q-PCR-R: $5^{\prime}$ :AGGGGAAATTCCGTTGAGTT-3'; BCL2 ChIP-q-PCR-F: 5'-ACTTTACATTTCTGTTGTGTTTACAGC-3', BCL2 ChIP-qPCR-R: $5^{\prime}$-ATTCATACATATGCACACGCACA- $3^{\prime}$; BCL-XL ChIP-q-PCR F: 5'-AATTCAGCTGCCAGCCTCT-3', BCL-XL ChIP-q-PCR R: 5'-CAACCGCTT CCTTTTCTGAG-3', HIF2 $\alpha$ (EPAS1) ChIP-q-PCR F: $5^{\prime}$-CAGTGTCCTGAGACT GTATG-3', HIF2 $\alpha$ (EPAS1) ChIP-q-PCR R: $5^{\prime}$-CTGTCAGACCCGAAAAGA-3', MIR155HG ChIP-q-PCR F: 5'-GAAAGGGAAAGGGGAAAACA-3', MIR155HG ChIP-q-PCR R: 5'-CGAACGTGCGACCCTTTTAT-3'.

AZD1480 treatment of mutant STAT3- or STAT5B-transduced KAI3 cells. In all, 25,000 KAI3 cells were seeded in $2 \mathrm{ml}$ inside 24-well plates in replicates or triplicates and treated with $0,0.5,1$ or $2 \mu \mathrm{M}$ of AZD1480 for $72 \mathrm{~h}$. Seventy-two hours post treatment, the total viable cell number in each well was quantified using a Vi-cell XR Cell Viability Analyzer (Beckman Coulter Inc.) according to the manufacturer's instructions. Total cell number in each treated sample was normalized to that of the untreated control cells.

Three-dimensional structural modelling of the STAT5B-N642H mutant. The STAT5B structure was modelled on the available STAT5A structure (PDB ID: $1 \mathrm{Y} 1 \mathrm{U})$ using the program MODELLER ${ }^{41}$. There was little difference in the SH2 domains as these two proteins have extremely similar primary sequences. Then, the modelled STAT5B SH2 domain was compared with other proteins containing SH2 domains that had been cocrystallized with peptides (v-SRC, GRB2, SH2B, NCK2) which showed the site of the $\mathrm{N} 642 \mathrm{H}$ was directly located in the key binding pocket of the phosphorylated tyrosine. Phosphorylated self-peptide (STAT5B: VDG-PTRVKPQ) was docked into the SH2 domain of WT-STAT5B or N642H STAT5B, 
respectively. Protein-protein docking was done with the FFT-based docking tool ClusPro $^{42}$ on a dedicated server. Key binding residues (STAT5B: R618, S620, N621, K600, N642) were specified based on previous peptide-SH2 domains cocrystallized structures (PDB ID: 2HDX, 1SHA, 2CIA, 1TZE). The best docking results were selected based on an electrostatically favoured scoring function. ClusPro docking server first clustered 1,000 ligand positions with the lowest energy score according to the 9 angstrom C-alpha RMSD radius and then ranked the best model. With the energy-minimized protein-peptide docked model, the binding energy of the complex was calculated with MolDock ${ }^{43}$.

Surface plasmon resonance binding assay. For surface plasmon resonance, WT and N642H-mutant STAT5B (residues between 128aa-717aa, NM_012448) was PCR cloned into the SMT3-pET28b + plasmid using the BamHI and XhoI cloning sites. BclI instead of BamHI restriction site was used in the forward primer to prevent digestion of STAT5B due to the presence of an internal BamHI site. A TGA stop codon was included in the reverse primer so that C-term His was not expressed. High-fidelity PfuUltra II Fusion HS DNA Polymerase (Agilent Technologies, Palo Alto, CA) was used to amplify STAT5B insert from STAT5B-WT-pMIG vector. Diagnostic mapping and Sanger sequencing of the inserts and the integration sites were performed to check the quality of the clones.

WT and mutant STAT5B expression was performed as follows: Plasmid DNA was transformed into BL21 (DE3) Codon Plus RIL competent cells (Agilent Technologies) and plated on LB agar plates with chloramphenicol (Cam) and kanamycin (Kan). Single colonies were selected and grown in LB media with Cam and Kan overnight at $37^{\circ} \mathrm{C}$. Overnight culture of $6 \times 6 \mathrm{ml}$ was used to inoculate $6 \times 11 \mathrm{LB}$ media with Kan and Cam. Cultures were grown at $37^{\circ} \mathrm{C}$ to an optical density of 0.6 , then flasks were moved to a precooled shaker at $18^{\circ} \mathrm{C}$. Cultures were grown at $18^{\circ} \mathrm{C}$ until they reached an optical density between 0.9 and 1.1 . Protein expression was induced with a final concentration of $500 \mu \mathrm{M}$ isopropyl- $\beta$-Dthiogalactoside, and cells were allowed to grow $16-20 \mathrm{~h}$ at $18^{\circ} \mathrm{C}$ with continued shaking. Cells were harvested by centrifugation, resuspended in PBS and frozen at $-20^{\circ} \mathrm{C}$ until purification.

His6-SMT fusions of both WT and N642 mutant STAT5B were purified as previously published ${ }^{44}$. Briefly, cells were thawed and lysed by French pressure cell with DNase I and PMSF. Lysates were clarified by centrifugation and filtration. Lysates were applied to Ni-NTA (Thermo Scientific HisPur) and washed with a PBS/imidazole gradient. Eluted protein was dialysed overnight at $4{ }^{\circ} \mathrm{C}$ into PBS in the presence of His6-ULP1 enzyme with $1 \mathrm{mM}$ dithiothreitol. The dialysed protein was incubated with Ni-NTA beads before concentration to remove uncleaved material, His6-SMT and His6-ULP1. The unbound material was then loaded onto the preparative grade superdex G200 gel filtration column (GE lifesciences) and exchanged into $50 \mathrm{mM}$ Tris $\mathrm{pH} 8.0,100 \mathrm{mM} \mathrm{NaCl}, 1 \mathrm{mM}$ EDTA, and $1 \mathrm{mM}$ dithiothreitol on column. The peak eluting at $\sim 190 \mathrm{ml}$ was concentrated, aliquotted into small volumes, and stored at $-80^{\circ} \mathrm{C}$.

Surface plasmon resonance studies were carried out with the GE Lifesciences Biacore T100 instrument at $25^{\circ} \mathrm{C}$. STAT5B-WT and N642H variant ligands were thawed and extensively dialysed into HBS-N buffer (GE Lifesciences). Protein samples of $50 \mu \mathrm{g} \mathrm{ml}^{-1}$ were made by diluting the dialysed stock samples into acetate $\mathrm{pH} 5.0$ buffer immediately before immobilization. Both STAT5B proteins were coupled using EDC/NHS amine coupling chemistry with final immobilization levels of 5345.6 RU for WT and 5433.9 RU for N642H variant. Reference channels received a blank amine coupling protocol. The analyte, phosphopeptide (KAVDG(p)YVKPQI) (Anaspec, Fremont, CA) was prepared by dissolution in water and extensive dialysis into water. The peptide stock solution was stored at $4{ }^{\circ} \mathrm{C}$ before analysis. Two-fold dilutions of the phosphopeptide stock were prepared in HBS-EP + (GE lifesciences) ranging from 100 to $0.78 \mu \mathrm{M}$ immediately before analysis. Peptide samples were flowed over the immobilized ligand at a rate of $30 \mu \mathrm{min}^{-1}$ with each concentration being run in duplicate. HBS-EP + was used both as running and regeneration buffer. $K_{\mathrm{D}}$ values were calculated using BiaEvaluation software (Biacore AS, Uppsala, Sweeden) by fitting the binding isotherms to a 1:1 Langmuir model.

Statistical analysis. Two-tailed unpaired $t$-test was applied using Microsoft Office Excel (Microsoft, Redmond, WA). $P<0.05$ was considered statistically significant.

\section{References}

1. Jaffe, E. S. The 2008 WHO classification of lymphomas: implications for clinical practice and translational research. Hematology Am. Soc. Hematol. Educ. Program 2009, 523-531 (2009).

2. Kwong, Y. L. Natural killer-cell malignancies: diagnosis and treatment. Leukemia 19, 2186-2194 (2005).

3. Takakuwa, T. et al. Frequent mutations of Fas gene in nasal NK/T cell lymphoma. Oncogene 21, 4702-4705 (2002).

4. Quintanilla-Martinez, L. et al. p53 Mutations in nasal natural killer/T-cell lymphoma from Mexico: association with large cell morphology and advanced disease. Am. J. Pathol. 159, 2095-2105 (2001).
5. Yu, H., Pardoll, D. \& Jove, R. STATs in cancer inflammation and immunity: a leading role for STAT3. Nat. Rev. Cancer 9, 798-809 (2009).

6. Koo, G. C. et al. Janus kinase 3 -activating mutations identified in natural killer/T-cell lymphoma. Cancer Discov. 2, 591-597 (2012).

7. Bouchekioua, A. et al. JAK3 deregulation by activating mutations confers invasive growth advantage in extranodal nasal-type natural killer cell lymphoma. Leukemia 28, 338-348 (2013).

8. Kimura, H. et al. Rare occurrence of JAK3 mutations in natural killer cell neoplasms in Japan. Leuk. Lymphoma 55, 962-963 (2014).

9. Iqbal, J. et al. Natural killer cell lymphoma shares strikingly similar molecular features with a group of non-hepatosplenic gammadelta T-cell lymphoma and is highly sensitive to a novel aurora kinase A inhibitor in vitro. Leukemia 25, 348-358 (2011).

10. Delabie, J. et al. Enteropathy-associated T-cell lymphoma: clinical and histological findings from the international peripheral $\mathrm{T}$-cell lymphoma project. Blood 118, 148-155 (2011).

11. Chan, J. K. et al. Type II enteropathy-associated T-cell lymphoma: a distinct aggressive lymphoma with frequent gammadelta $\mathrm{T}$-cell receptor expression. Am. J. Surg. Pathol. 35, 1557-1569 (2011).

12. Kanai, T. et al. Identification of STAT5A and STAT5B target genes in human T cells. PLoS ONE 9, e86790 (2014).

13. Silva, M. et al. Erythropoietin can induce the expression of bcl- $\mathrm{x}(\mathrm{L})$ through Stat5 in erythropoietin-dependent progenitor cell lines. J. Biol. Chem. 274, 22165-22169 (1999).

14. Li, G. et al. STAT5 requires the N-domain for suppression of miR15/16, induction of bcl-2, and survival signaling in myeloproliferative disease. Blood 115, 1416-1424 (2010).

15. Kopp, K. L. et al. STAT5-mediated expression of oncogenic miR-155 in cutaneous T-cell lymphoma. Cell Cycle 12, 1939-1947 (2013).

16. Fatrai, S., Wierenga, A. T., Daenen, S. M., Vellenga, E. \& Schuringa, J. J. Identification of HIF2alpha as an important STAT5 target gene in human hematopoietic stem cells. Blood 117, 3320-3330 (2011).

17. Nagy, Z. S. et al. STAT5 regulation of BCL10 parallels constitutive NFkappaB activation in lymphoid tumor cells. Mol. Cancer 8, 67 (2009).

18. Johnston, J. A., Bacon, C. M., Riedy, M. C. \& O'Shea, J. J. Signaling by IL-2 and related cytokines: JAKs, STATs, and relationship to immunodeficiency. J. Leukoc. Biol. 60, 441-452 (1996).

19. Johnston, J. A. et al. Tyrosine phosphorylation and activation of STAT5, STAT3, and Janus kinases by interleukins 2 and 15. Proc. Natl Acad. Sci. USA 92, 8705-8709 (1995).

20. Derenzini, E. et al. The JAK inhibitor AZD1480 regulates proliferation and immunity in Hodgkin lymphoma. Blood Cancer J. 1, e46 (2011).

21. Pardanani, A. JAK2 inhibitor therapy in myeloproliferative disorders: rationale, preclinical studies and ongoing clinical trials. Leukemia 22, 23-30 (2008).

22. Sonbol, M. B. et al. Comprehensive review of JAK inhibitors in myeloproliferative neoplasms. Ther. Adv. Hematol. 4, 15-35 (2013).

23. Ohgami, R. S., Ma, L., Monabati, A., Zehnder, J. L. \& Arber, D. A. STAT3 mutations are present in aggressive B-cell lymphomas including a subset of diffuse large B-cell lymphomas with CD30 expression. Haematologica 99, e105-e107 (2014).

24. Odejide, O. et al. A targeted mutational landscape of angioimmunoblastic T-cell lymphoma. Blood 123, 1293-1296 (2014).

25. Ohgami, R. S. et al. STAT3 mutations are frequent in CD30 + T-cell lymphomas and T-cell large granular lymphocytic leukemia. Leukemia 27, 2244-2247 (2013).

26. Jerez, A. et al. STAT3 mutations unify the pathogenesis of chronic lymphoproliferative disorders of NK cells and T-cell large granular lymphocyte leukemia. Blood 120, 3048-3057 (2012).

27. Koskela, H. L. et al. Somatic STAT3 mutations in large granular lymphocytic leukemia. New Engl. J. Med. 366, 1905-1913 (2012).

28. Rajala, H. L. et al. Discovery of somatic STAT5b mutations in large granular lymphocytic leukemia. Blood 121, 4541-4550 (2013).

29. Kiel, M. J. et al. Integrated genomic sequencing reveals mutational landscape of T-cell prolymphocytic leukemia. Blood 124, 1460-1472 (2014).

30. Iqbal, J. et al. Genomic analyses reveal global functional alterations that promote tumor growth and novel tumor suppressor genes in natural killer-cell malignancies. Leukemia 23, 1139-1151 (2009).

31. Nagata, H. et al. Characterization of novel natural killer (NK)-cell and gammadelta $\mathrm{T}$-cell lines established from primary lesions of nasal T/NK-cell lymphomas associated with the Epstein-Barr virus. Blood 97, 708-713 (2001).

32. Schmitz, R. et al. Burkitt lymphoma pathogenesis and therapeutic targets from structural and functional genomics. Nature 490, 116-120 (2012).

33. Abecasis, G. R. et al. An integrated map of genetic variation from 1,092 human genomes. Nature 491, 56-65 (2012).

34. Li, H. \& Durbin, R. Fast and accurate short read alignment with BurrowsWheeler transform. Bioinformatics 25, 1754-1760 (2009). 
35. DePristo, M. A. et al. A framework for variation discovery and genotyping using next-generation DNA sequencing data. Nat. Genet. 43, 491-498 (2011).

36. Wang, K., Li, M. \& Hakonarson, H. ANNOVAR: functional annotation of genetic variants from high-throughput sequencing data. Nucleic Acids Res. 38, e164 (2010).

37. Kucuk, C. et al. HACE1 is a tumor suppressor gene candidate in natural killer cell neoplasms. Am. J. Pathol. 182, 49-55 (2013).

38. Somanchi, S. S., Senyukov, V. V., Denman, C. J. \& Lee, D. A. Expansion, purification, and functional assessment of human peripheral blood NK cells. J. Vis. Exp. e2540 (2011).

39. Kucuk, C. et al. PRDM1 is a tumor suppressor gene in natural killer cell malignancies. Proc. Natl Acad. Sci. USA 108, 20119-20124 (2011).

40. Piva, R. et al. Gene expression profiling uncovers molecular classifiers for the recognition of anaplastic large-cell lymphoma within peripheral T-cell neoplasms. J. Clin. Oncol. 28, 1583-1590 (2010).

41. Sali, A. \& Blundell, T. L. Comparative protein modelling by satisfaction of spatial restraints. J. Mol. Biol. 234, 779-815 (1993).

42. Comeau, S. R., Gatchell, D. W., Vajda, S. \& Camacho, C. J. ClusPro: an automated docking and discrimination method for the prediction of protein complexes. Bioinformatics 20, 45-50 (2004).

43. Thomsen, R. \& Christensen, M. H. MolDock: a new technique for highaccuracy molecular docking. J. Med. Chem. 49, 3315-3321 (2006).

44. Donaldson, J. M. et al. Identification and grafting of a unique peptide-binding site in the Fab framework of monoclonal antibodies. Proc. Natl Acad. Sci. USA 110, 17456-17461 (2013).

\section{Acknowledgements}

We thank Dr Dean A. Lee for the K562-Clone9-mb21 cell line and Dr Giorgio Inghirami (NYU Langone Medical Center) for providing the PLVTH-S3S construct.

\section{Author contributions}

C.K. designed and performed the experiments, analysed the data and wrote the manuscript; B.J. and X.H. performed the research, analysed the data; Wenyan Z., J.K.C.C., P.G., Q.Y., H.S., F.D., S.L., W.L., L.L. provided and characterized tumour samples; J.C.W., K.N.A. performed SPR analysis; A.S., L.S., J.I., K.F.,T.M. assisted in the design of the experiments; W.X., P.K., C.A., Weiwei Z., A.C., Q.G.,T.M analysed RNA-Seq and WES data; N.L. and E.S. analysed the three-dimensional structural model; W.C.C. conceived and supervised the project, and edited the manuscript.

\section{Additional information}

Accession codes. The RNA-Seq and WES sequencing data have been deposited into the SRA database (http://www.ncbi.nlm.nih.gov/sra), under the accession code SRA200820.

Supplementary Information accompanies this paper at http://www.nature.com/ naturecommunications

Competing financial interests: The authors declare no competing financial interests.

Reprints and permission information is available online at http://npg.nature.com/ reprintsandpermissions/

How to cite this article: Küçük, C. et al. Activating mutations of STAT5B and STAT3 in lymphomas derived from $\gamma \delta$-T or NK cells. Nat. Commun. 6:6025 doi: $10.1038 /$ ncomms7025 (2015). 